\title{
Bevacizumab plus chemotherapy continued beyond progression in patients with type II endometrial cancer previously treated with bevacizumab plus chemotherapy: A case report
}

\author{
EMI SATO, KENTARO NAKAYAMA, KOHEI NAKAMURA, TOMOKA ISHIBASHI, \\ HIROSHI KATAGIRI, MASAKO ISHIKAWA and SATORU KYO
}

Department of Obstetrics and Gynecology, Shimane University School of Medicine, Izumo, Shimane 693-8501, Japan

Received September 14, 2015; Accepted February 28, 2017

DOI: $10.3892 / \mathrm{mco} .2017 .1316$

\begin{abstract}
The prognosis of patients with recurrent/persistent endometrial cancer, particularly type II cancer, remains poor, and effective treatment has not yet been established. We herein present the case of a patient with recurrent type II endometrial cancer who received bevacizumab + chemotherapy continued beyond progression, after previously receiving bevacizumab + chemotherapy. This patient experienced recurrence after firstand second-line adjuvant chemotherapy followed by modified radical hysterectomy and she was administered bevacizumab + paclitaxel + carboplatin therapy. After six cycles of treatment, all metastatic lesions shrunk, indicating partial response. The patient next received single-agent bevacizumab as maintenance therapy. After 12 cycles of bevacizumab monotherapy, disease progression was detected; therefore, combination therapy consisting of bevacizumab, doxorubicin and carboplatin was initiated. After six cycles of this combination therapy, the patient exhibited disease stabilization. Finally, 18 months after the initial bevacizumab treatment, the patient remained on combination chemotherapy, without complaints or signs of tumor progression (last follow-up, October 2014).
\end{abstract}

\section{Introduction}

Endometrial cancer is a common gynecological malignancy in western countries, whereas its incidence has also been increasing in Asia (1). Endometrial cancer may be subdivided into types I and II. Type I tumors, which are associated with obesity or exogenous estrogen use, present at an early stage and have an excellent prognosis. Histologically, these tumors are well- to moderately-differentiated endometrioid adenocarcinomas, with typically minimal invasion and infrequent

Correspondence to: Dr Kentaro Nakayama, Department of Obstetrics and Gynecology, Shimane University School of Medicine, Enyacho 89-1, Izumo, Shimane 693-8501, Japan

E-mail:kn88@med.shimane-u.ac.jp

Key words: endometrial cancer, bevacizumab, molecular-targeted drugs, chemotherapy, recurrence nodal metastasis. Type II tumors are not classically associated with obesity or exogenous estrogen use. Histologically, these tumors are poorly differentiated endometrioid carcinomas, with serous or clear-cell characteristics (2). The standard chemotherapy for endometrial cancer worldwide currently consists of a combination of doxorubicin and cisplatin (AP therapy). Combinations of taxanes and platinum-containing drugs (TC therapy) have also been used as adjuvant therapy and as chemotherapy for advanced or recurrent endometrial cancer (3). However, the prognosis of patients with metastatic disease remains disappointing, with an overall survival of only 1 year commonly reported, despite treatment efforts (4).

Currently, genetic abnormalities associated with the onset and progression of cancer have been identified in the endometrial cell membrane and signaling systems, and the developmental mechanisms of endometrial cancer are gradually becoming elucidated. The identification of the molecules associated with these abnormalities has led to new potential treatment regimens for endometrial cancer using molecularly-targeted agents (3). For example, preclinical models have demonstrated the efficacy of bevacizumab in combination with chemotherapy against endometrial cell lines (4). In clinical practice, the GOG phase II trial is the most recent completed trial of targeted therapy in recurrent/persistent endometrial cancer (5). We hypothesized that bevacizumab may be active in endometrial cancer. We herein present the case of a patient with recurrent endometrial cancer who experienced a prolonged response to combination treatment with bevacizumab + chemotherapy.

\section{Case report}

A 78-year-old woman, gravida 3, para 2, was referred to the Shimane University Hospital (Izumo, Japan) for examination and treatment of a pelvic mass. The carbohydrate antigen (CA)-125 level was $32 \mathrm{ng} / \mathrm{ml}$. No metastatic lesions were identified. The patient underwent modified radical hysterectomy, bilateral salpingo-oophorectomy, pelvic lymph node dissection and omentectomy. Pathological examination revealed a stage IIA (International Federation of Obstetrics and Gynecology 1988) endometrioid adenocarcinoma, grade 2 endometrial cancer (myometrial invasion $40 \%$, ascitic fluid 
A
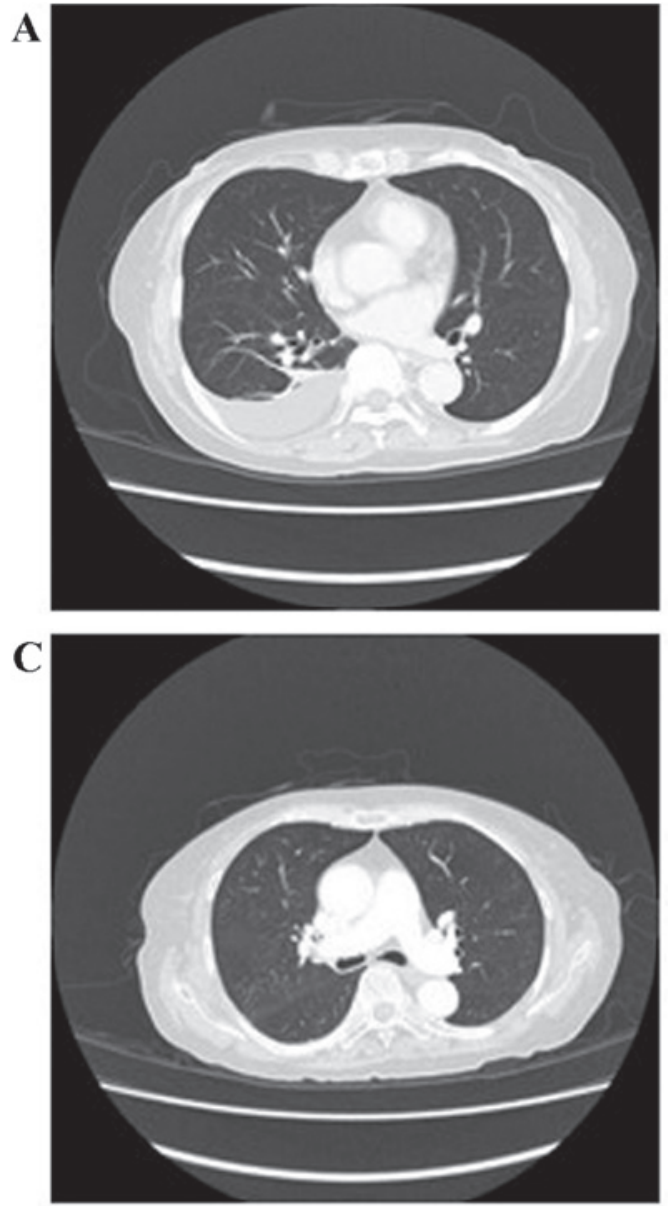

B

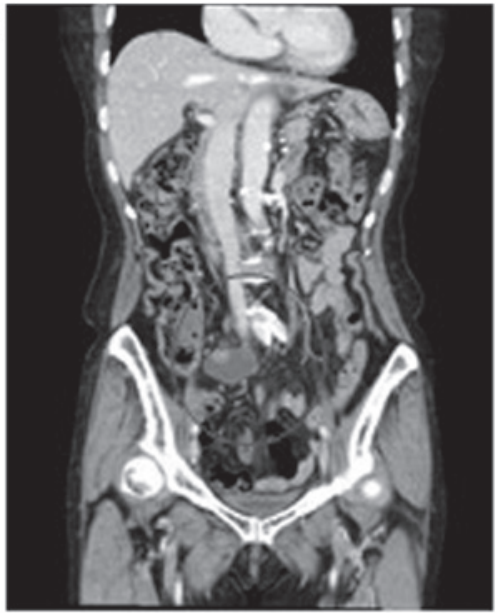

D

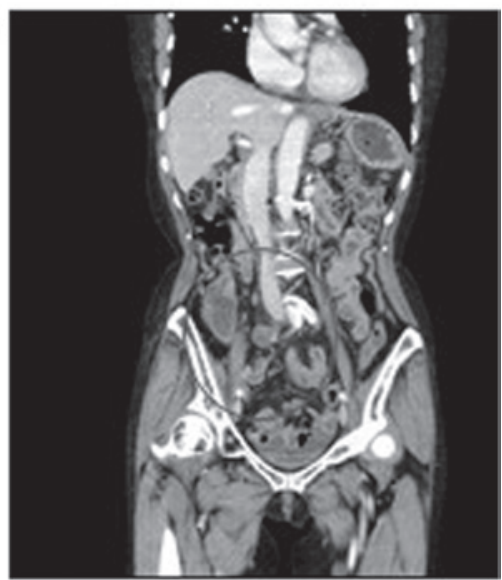

Figure 1. Computed tomography images of a patient with recurrent endometrial cancer. Progressive disease following taxane and platinum (TC) therapy: (A) Pleural effusion and (B) para-aortic lymph node metastasis. After 6 cycles of combination therapy with TC and bevacizumab, the patient achieved partial response: (C) Pleural effusion and (D) para-aortic lymph node metastasis.

cytology-positive, estrogen receptor-negative, progesterone receptor-negative and p53 mutation-positive). The immunohistochemistry results confirmed the diagnosis of type II endometrial cancer.

As adjuvant therapy, the patient received combination chemotherapy consisting of paclitaxel $\left(135 \mathrm{mg} / \mathrm{m}^{2}\right)$ and carboplatin [area under the curve (AUC)=4] (TC therapy). Due to impaired hepatic function, chemotherapy was discontinued after three cycles.

Eleven months after adjuvant chemotherapy, the CA1-25 level started to increase; pleural metastasis, pelvic lymph node metastasis, pleural effusion and ascites were observed on computed tomography (CT) examination. The patient was diagnosed with recurrence of endometrial cancer. After recovery of hepatic function was confirmed, she received three cycles of TC therapy. Unfortunately, the pleural and pelvic lymph node metastasis progressed, with detection of intraperitoneal implantations and para-aortic lymph node metastasis (Fig. 1A and B). The patient was then initiated on combination chemotherapy consisting of adriamycin $\left(60 \mathrm{mg} / \mathrm{m}^{2}\right)$ and cisplatin $\left(50 \mathrm{mg} / \mathrm{m}^{2}\right.$ ) (AP therapy). After two cycles, an increase in the amount of pleural effusion was observed. Although both the TC and AP regimens were used, the CA-125 levels continued to increase and the $\mathrm{CT}$ images suggested progressive disease. The Ethics Committee of the hospital granted approval for the

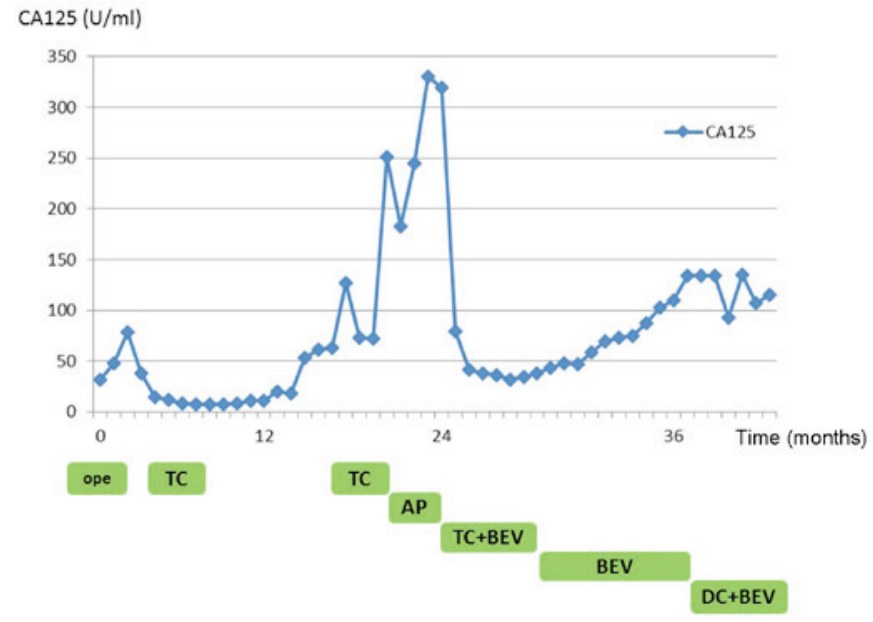

Figure 2. Response to bevacizumab therapy. This graph shows the carbohydrate antigen (CA)-125 biomarker level response to chemotherapy + bevacizumab in the present case. TC, taxane and platinum; AP, doxorubicin and cisplatin; BEV, bevacizumab; DC, doxorubicin and carboplatin.

use of bevacizumab to treat the recurrent and persistent endometrial cancer, and treatment was initiated after obtaining written informed consent from the patient.

First, combination therapy consisting of paclitaxel $\left(175 / \mathrm{m}^{2}\right)$, carboplatin $(\mathrm{AUC}=3.5)$ and bevacizumab $(15 \mathrm{mg} / \mathrm{kg})$ 
$(\mathrm{TC}+\mathrm{BEV})$ was initiated and administered every 3 weeks After two cycles of TC + BEV therapy, the CA-125 level started to decrease. After six cycles of therapy, all the metastatic lesions had shrunk, indicating partial response according to the Response Evaluation Criteria in Solid Tumors (RECIST), version 1.1 (http://www.jcog.jp/doctor/tool/C_150_0010. pdf); the pleural effusion and ascites also disappeared (Fig. 1C and D). Next, based on the regimen used in ovarian cancer, the patient received single-agent bevacizumab as maintenance therapy. After 12 cycles of bevacizumab monotherapy (8 months after initiating TC $+\mathrm{BEV}$ ), the CA-125 level started to increase, and intraperitoneal implantations were once again detected; thus, combination therapy consisting of doxorubicin $\left(70 \mathrm{mg} / \mathrm{m}^{2}\right)$, carboplatin $(\mathrm{AUC}=3.5)$ and bevacizumab $(15 \mathrm{mg} / \mathrm{kg})(\mathrm{DC}+\mathrm{BEV})$ was initiated. During bevacizumab therapy, the patient developed grade 2 peripheral neuropathy and deep venous thrombosis; no grade $>2$ toxicities were observed. Six cycles after the initiation of DC + BEV therapy, the patient exhibited no newly disseminated tumors, pleural effusion, or ascites. At 18 months after the initial bevacizumab treatment (last follow-up, October 2014), the patient remained on combination chemotherapy, without complaints or signs of tumor progression (Fig. 2).

\section{Discussion}

The prognosis of patients with recurrent/persistent endometrial cancer remains disappointing, and an effective treatment has not yet been established. Currently, bevacizumab is reported to be effective against newly diagnosed as well as recurrent epithelial ovarian cancer (6-8). Furthermore, the addition of bevacizumab to combination chemotherapy in patients with recurrent, persistent or metastatic cervical cancer has been previously reported to improve the median overall survival (9). However, for recurrent/persistent endometrial cancer, the GOG phase II trial is the most recently reported trial of bevacizumab monotherapy (5).

This patient experienced a significant reduction of both the disseminated tumors and ascites. Reduction of ascites may be explained by inhibition of the vascular endothelial growth factor pathway, as bevacizumab has the ability to suspend ascitic fluid production resulting from peritoneal dissemination in solid cancers. Of note, the disseminated tumors in the present case were also reduced. By combining bevacizumab and chemotherapy, the effects of each drug may have been enhanced.

The patient continued to receive bevacizumab, and when disease progression was observed, bevacizumab + chemotherapy was again initiated, after which time the patient experienced a long-term response. It is possible that the anti-angiogenic effects of bevacizumab require the continued presence of the drug to effectively suppress the growth of minimal residual disease. Our patient, who experienced a prolonged response during bevacizumab therapy, may represent a group of patients that are particularly sensitive to this agent, and who may respond to a bevacizumab rechallenge at the time of relapse. Relapse following bevacizumab withdrawal may not always represent resistance to this agent, but rather the release of anti-angiogenic selection pressure that may occasionally be restored by reinstituting the agent (6).
The cytotoxicity of current anticancer drugs also applies to normal cells, and the achievement of therapeutic efficacy is often associated with severe adverse events. Chemotherapy must be performed while considering the balance between therapeutic benefits and adverse effects. By contrast, molecularly-targeted drugs act by specifically targeting the mechanisms of cancer cell proliferation and metastasis at the molecular level. The targeting of specific molecules in cancer cells may achieve therapeutic efficacy with fewer side effects (3). In fact, the patient in the present case did not experience grade $>2$ toxicities throughout the bevacizumab treatment.

The findings in this case suggest that bevacizumab may be effective against recurrent/persistent endometrial cancer. In particular, patients with type II endometrial cancer, which is less sensitive to chemotherapy, may benefit from the use of bevacizumab. Our patient experienced a partial response according to RECIST, and a significant decrease of the CA-125 level as a result of bevacizumab treatment. The patient repeatedly experienced disease progression while on bevacizumab monotherapy, but then responded following reinitiation of combination bevacizumab/chemotherapy, suggesting that the anti-angiogenic action of bevacizumab reduced tumor neovascularization, promoting permeation of the tumor by the chemotherapy agent through the remaining vessels. To the best of our knowledge, this case is the first report of bevacizumab + chemotherapy administered following disease progression in a patient with type II endometrial cancer previously treated with bevacizumab + chemotherapy.

A recent phase II trial of a paclitaxel, carboplatin and bevacizumab regimen that is effective and tolerable in advanced and recurrent endometrial cancer was reported (10). The patient in the present case also experienced a partial response to combination bevacizumab + chemotherapy and an unusually long duration of response. Taken together, the recent report and the present case suggest that a taxane/platinum + bevacizumab regimen may be a viable therapeutic option for the treatment of endometrial cancer.

Given the lack of effective therapies for patients with recurrent/persistent endometrial cancer, this case suggests that prospective randomized clinical trials of combination bevacizumab + chemotherapy regimens in endometrial cancer may be required. Furthermore, the present case also suggested that bevacizumab + chemotherapy may be an effective treatment option for patients with endometrial cancer who previously received bevacizumab + standard chemotherapy.

\section{Acknowledgements}

The authors would like to thank the medical staff of the Shimane University Hospital.

\section{References}

1. Siegel R, Ma J, Zou Z and Jemal A: Cancer statistics, 2014. CA Cancer J Clin 64: 9-29, 2014.

2. Bokhman JV: Two pathogenetic types of endometrial carcinoma. Gynecol Oncol 15: 10-17, 1983.

3. Nogami Y, Banno K, Kisu I, Yanokura M, Umene K, Masuda K, Kobayashi Y, Yamagami W, Nomura H, Tominaga E, et al: Current status of molecular-targeted drugs for endometrial cancer (Review). Mol Clin Oncol 1: 799-804, 2013. 
4. Zaqouri F, Bozas G, Kafantari E, Tsiatas M, Nikitas N, Dimopoulos MA and Papadimitriou CA: Endometrial cancer: What is new in adjuvant and molecularly targeted therapy? Obstet Gynecol Int 2010: 749579, 2010.

5. Aqhajanian C, Sill MW, Darcy KM, Greer B, McMeekin DS Rose PG, Rotmensch J, Barnes MN, Hanjani P and Leslie KK: Phase II trial of bevacizumab in recurrent or persistent endometrial cancer: A Gynecologic Oncology Group Study. J Clin Oncol 29: 2259-2265, 2011.

6. Konstantinopoulos PA, Berlin ST, Campos SM, Matulonis UA and Cannistra SA: Bevacizumab rechallenge after first line maintenance bevacizumab. Gynecol Oncol 125: 510-511, 2012.

7. Pujade-Lauraine E, Hilpert F, Weber B, Reuss A, Poveda A, Kristensen G, Sorio R, Vergote I, Witteveen P, Bamias A, et al: Bevacizumab combined with chemotherapy for platinum-resistanct recurrent ovarian cancer: The AURELIA open-label randomized phase III trial. J Clin Oncol 32: 1302-1308, 2014.
8. Aqhajanian C, Blank SV, Goff BA, Judson PL, Teneriello MG, Husain A, Sovak MA, Yi J and Nycum LR: OSEANS: A randomized, double-blind, placebo-controlled phase III trial of chemotherapy with or without bevacizumab in patients with platinum-sensitive recurrent epithelial ovarian, primary peritoneal, or fallopian tube cancer. J Clin Oncol 30: 2039-2045, 2012.

9. Tewari KS, Sill MW, Long HJ III, Penson RT, Huang H, Ramondetta LM, Landrum LM, Oaknin A, Reid TJ, Leitao MM, et al: Improved survival with bevacizumab in advanced cervical cancer. N Engl J Med 370: 734-743, 2014.

10. Simpkins F, Drake R, Escobar PF, Nutter B, Rasool N and Rose PG: A phase II trial of paclitaxel, carboplatin, and bevacizumab in advanced and recurrent endometrial carcinoma (EMCA). Gynecol Oncol 136: 240-245, 2015. 